\title{
Lung volume reduction surgery or bronchoscopic lung volume reduction: is there an algorithm for allocation?
}

\author{
Matthew Gordon, Sean Duffy, Gerard J. Criner \\ Department of Thoracic Medicine and Surgery, Lewis Katz School of Medicine at Temple University, Philadelphia, PA, USA \\ Contributions: (I) Conception and design: All authors; (II) Administrative support: M Gordon, S Duffy; (III) Provision of study materials or patients: \\ All authors; (IV) Collection and assembly of data: All authors; (V) Data analysis and interpretation: All authors; (VI) Manuscript writing: All authors; \\ (VII) Final approval of manuscript: All authors. \\ Correspondence to: Matthew Gordon, MD. Department of Thoracic Medicine and Surgery, Lewis Katz School of Medicine at Temple University, \\ Philadelphia, PA, USA. Email: Matthew.Gordon@tuhs.temple.edu.
}

\begin{abstract}
Chronic obstructive pulmonary disease (COPD) is the fourth leading cause of death in the world with the annual number of deaths increasing every year. Alterations in chest wall mechanics, respiratory muscle mechanics, and impaired cardiac function that result from increased air-trapping are well known sequelae of COPD and contribute to increased morbidity and mortality. A reduction in hyperinflation can improve cardiopulmonary function. In selected patients with COPD and an emphysema phenotype, lung volume reduction surgery (LVRS) has demonstrated improvements in symptom burden and mortality. Minimally invasive bronchoscopic techniques that reduce end-expiratory lung volume have shown improvements in lung function, dyspnea and quality of life. In this review, we review selection criteria, risks, and benefits of surgical and bronchoscopic lung volume reduction (BLVR). Recommendations are provided to guide treatment decisions based on the current literature.
\end{abstract}

Keywords: Lung volume reduction surgery (LVRS); bronchoscopic lung volume reduction (BLVR); hyperinflation; chronic obstructive pulmonary disease (COPD); endobronchial coil; endobronchial valve (EBV)

Submitted Nov 26, 2017. Accepted for publication May 15, 2018.

doi: $10.21037 /$ jtd.2018.05.118

View this article at: http://dx.doi.org/10.21037/jtd.2018.05.118

\section{Introduction}

Chronic obstructive pulmonary disease (COPD) is a disease with significant morbidity and mortality. Currently the fourth leading cause of death, COPD has an increasing number of deaths per year, and is projected to be the third leading cause of death by $2020(1,2)$. While symptomatic treatment options for COPD have expanded, therapies that impact mortality are still limited to smoking cessation (3), supplemental oxygen (in those with significant hypoxemia) $(4,5)$, and lung volume reduction surgery (LVRS) in appropriately selected patients (6).

The potential harms associated with hyperinflation, both static and dynamic, have been recognized for decades. Lung hyperinflation alters chest wall mechanics, placing respiratory muscles at a mechanical disadvantage.
Hyperinflation increases the sensation of dyspnea as well as objectively decreasing exercise capacity. In addition to pulmonary mechanics, research shows that cardiac function is also impaired due to increased intrathoracic pressure and decreased cardiac chamber filling.

Reduction in hyperinflation has been shown to improve function of the cardiopulmonary unit, increase exercise capacity, decrease dyspnea, and in a select patient population, decrease mortality. This was never more evident than in the National Emphysema Treatment Trial (NETT), one of the largest and most comprehensive randomized controlled trials (RCTs) in severe emphysema patients (6). NETT was instrumental in highlighting the importance of physiological and radiographic phenotyping of COPD, and using these subgroups to predict mortality and response to treatment. 
Table 1 Key inclusion and exclusion criteria from NETT

\begin{tabular}{ll}
\hline Inclusion criteria & Exclusion criteria \\
\hline $\begin{array}{l}\mathrm{FEV}_{1} \leq 45 \% \text { (FEV } \\
\text { if age }>70 \text { years) }\end{array}$ & $\begin{array}{l}\mathrm{FEV}_{1} \leq 20 \% \text { and homogenous } \\
\text { emphysema or DLCO } \leq 20 \%\end{array}$ \\
$\mathrm{TLC} \geq 100 \%$ & Prior sternotomy, LVRS, or lobectomy \\
$\mathrm{RV} \geq 150 \%$ & Bulla $\geq 1 / 3 \mathrm{rd}$ of lung \\
Completion of & $\mathrm{LVEF} \leq 45 \%$ or $\mathrm{Ml}$ within the prior \\
pulmonary rehab & 6 months \\
program & \\
Nonsmoker for $\geq$ & Prednisone $\geq 20$ mg/day; pulmonary \\
4 months & hypertension; $6-\mathrm{MWD}<140$ meters \\
\hline
\end{tabular}

NETT, National Emphysema Treatment Trial; DLCO, diffusion capacity for the lungs using carbon monoxide; $F V_{1}$, forced expiratory volume in one second; LVEF, left ventricular ejection fraction; LVRS, lung volume reduction surgery; MI, myocardial infarction; RV, residual volume; TLC, total lung capacity; 6-MWD, 6-minute walk distance.

Despite a clear mortality benefit and durable improvement in symptom scores following LVRS, the number of LVRS surgeries performed has declined significantly (7). It remains unclear whether this decline is due to the increased mortality noted in certain subgroups, perioperative morbidity, or costs (7).

Following the NETT study that clearly demonstrated improvements in symptoms and mortality, multiple minimally invasive techniques have emerged. These include the use of one-way endobronchial valves (EBVs), endobronchial nitinol coils, foam sealant, and thermal ablation. These bronchoscopic techniques all attempt to provide comparable improvements in function via lung reduction but with reduced perioperative morbidity and mortality that is associated with traditional surgical approaches.

Despite advances in medical and interventional techniques, COPD remains a complex, heterogeneous disease with significant morbidity and mortality. As we enter the age of personalized medicine, interventional techniques need to be refined and tailored to specific patients with clearly defined pathophysiologic processes and specific phenotypes.

\section{Historical background of LVR}

Early surgical interventions that attempted to treat hyperinflation focused on altering the size and shape of the thoracic cavity to accommodate hyper-inflated lungs.
Lung volume reduction was attempted as early as the 1950 s, with Brantigan often credited with the first attempts at surgical lung volume reduction (8). However, this approach was not widely adopted due to an increased operative mortality of $18 \%$ and a lack of documented post-operative improvements. Interest in LVRS was reinvigorated in 1995, when Cooper published a case series of 20 patients that showed significant improvement in lung function and 6-minute walk distance (6-MWD) (9). This series was followed by a larger, 150 patient series by the same group, with the benefits of LVRS confirmed by the first RCT in 1999 by Criner et al. $(10,11)$ This was quickly followed by a second RCT with similar outcomes (12).

Following widespread adoption of LVRS, clinical outcomes were significantly worse than previously published research outcomes, with a reported mortality rate of $26 \%$. This discordance resulted in the NETT trial, a prospective, multicenter, RCT that compared optimal medical therapy to optimal medical therapy plus LVRS (6).

The NETT study enrolled a highly select, comprehensively characterized group of patients, screening 3,777 patients and randomizing 1,218 (Table 1). Primary endpoints included mortality and changes in exercise capacity $(>10 \mathrm{~W})$, with secondary endpoints that included changes in lung function, symptom scores, and quality of life assessments. Importantly, all patients underwent rigorous medical optimization prior to enrollment, including mandatory pulmonary rehabilitation. Patients with significant cardiac comorbidities that would preclude surgery were excluded from the study.

\section{Surgical lung volume reduction}

\section{LVRS}

\section{Patient selection}

When considering invasive interventions for patients with advanced COPD, detailed characterization is paramount. Pulmonary function testing (PFT), maximal cardiopulmonary exercise tests (CPETs), high resolution computerized tomography (HRCT), as well as perfusion scintigraphy are necessary to quantify operative risk and anticipated benefit (Table 2). Preoperative testing should be undertaken after medical optimization, including pulmonary rehabilitation. Forced expiratory volume of one second $\left(\mathrm{FEV}_{1}\right)$ and diffusion capacity of carbon monoxide (DLCO) are key inclusion and exclusion parameters used to determine eligibility for surgery and/or bronchoscopic 
Table 2 Outcomes of NETT study based on distribution of emphysema and exercise capacity

\begin{tabular}{lccccc}
\hline \multirow{2}{*}{ Parameter } & \multicolumn{2}{c}{ Homogeneous emphysema } & \multicolumn{2}{c}{ Upper-lobe emphysema } \\
\cline { 2 - 3 } Mortality & Increased & No change & No change* & Decreased \\
Exercise capacity & No change & No change & Increased & Increased \\
Symptoms & No change & Short term improvement & Improved & Improved \\
FEV ${ }_{1}<20 \%$ and DLCO $<20 \%$ or & & & Increased mortality & \\
homogeneous emphysema & & & & & \\
\hline
\end{tabular}

High exercise capacity is defined as $>25 \mathrm{~W}$ for women and $>40 \mathrm{~W}$ for men, as measured on post-pulmonary rehab CPET. Exercise capacity defined as a $10 \mathrm{~W}$ improvement on CPET. Symptom improvement defined as 8+ change in SGRQ. *, patients with a decreased diffusion capacity in the upper lobes may have a mortality benefit. NETT, National Emphysema Treatment Trial; FEV ${ }_{1}$, forced expiratory volume in one second; DLCO, diffusion capacity for the lungs using carbon monoxide; SGRQ, St. George's respiratory questionnaire; CPET, cardiopulmonary exercise test.

reduction. With regard to surgical classification, exercise capacity differs by sex, following the predefined criteria from the NETT study. High exercise capacity is defined as $>25 \mathrm{~W}$ for women and $>40 \mathrm{~W}$ for men, as measured on post-pulmonary rehab CPET.

\section{Surgical patients at increased risk of death}

Patients with a $\mathrm{FEV}_{1} \leq 20 \%$ and either a DLCO $\leq 20 \%$ or homogeneous emphysema noted on HRCT carry an excessive operative risk compared to medical treatment alone. In the NETT study, 30-day mortality in this group of patients was $16 \%$, compared to $0 \%$ in the medical arm. Patients in this group who survived surgery were unlikely to have a significant change in either exercise capacity or quality of life. Alternatives to lung volume reduction such as lung transplantation or medical therapy alone should be considered in this patient population.

Among patients with a high exercise tolerance and a non-upper lobe distribution of emphysema, LVRS is also associated with an increase in mortality when compared to medical therapy alone. These patients should not be offered LVRS. Given high exercise capacity, optimal medical therapy in conjunction with assessment for bronchoscopic lung volume reduction (BLVR) should be undertaken.

\section{Surgical patients without durable improvement}

Patients with non-upper lobe predominant disease and a low exercise capacity who underwent LVRS experienced a significant decrease in their symptoms. However, when compared to medically treated patients, there was no statistically significant change in symptom score by year 3. There was no improvement in exercise capacity or survival benefit in LVRS patients. Given the lack of durable improvement in symptom scores and high exercise capacity and mortality rates, patients in this subset of patients should not undergo LVRS.

When viewed as a whole, patients with a homogeneous distribution of emphysema receive minimal benefit, and in some cases, increased risk of death. LVRS should not be considered in patients without upper-lobe predominant emphysema.

\section{Surgical patients with durable improvement}

Patients with upper-lobe predominant distribution of disease as well as $\mathrm{FEV}_{1}>20 \%$ and DLCO $>20 \%$ should be considered for LVRS. In this subset of patients, those with a low exercise capacity experienced a significant improvement in mortality (risk ration $0.47, \mathrm{P}=0.005$ ), a significant increase in exercise capacity $(30 \%$ vs. $0 \%, \mathrm{P}<0.001)$, and an improvement in symptoms. These improvements in mortality, exercise capacity, and symptom score remained significant in both short and long-term follow-up at 3 years. Among all of the invasive procedures available to patients with COPD, this is the only intervention with a clearly proven mortality benefit. LVRS is strongly recommended in this patient population, unless comorbidities preclude surgical intervention.

When taken as group, high exercise capacity patients with upper-lobe disease did not accrue a mortality benefit, either short or long-term, when compared to those who underwent conservative medical therapy. However, high exercise capacity patients with decreased perfusion as 
measured by perfusion scintigraphy in the upper lobes did have a decrease in mortality, similar to the low exercise capacity group (13). This subset of high exercise capacity patients should be considered for LVRS.

In addition to mortality benefits in a subset of high exercise capacity patients, there was a statistically significant increase in exercise capacity $(15 \%$ vs. $3 \%, \mathrm{P}<1.001)$ and symptom score $(41 \%$ vs. $11 \%, \mathrm{P}<0.001)$ in those who underwent LVRS. These improvements in symptoms and exercise capacity persisted through all 3 years of followup. Given the high cost and perioperative morbidity, LVRS is not frequently recommended in this patient population. However, in patients with pulmonary nodules undergoing upper-lobe thoracic surgery, LVRS may be considered. Pulmonary nodules requiring follow-up are a relative contraindication to BLVR as atelectasis and parenchymal changes limit CT follow-up.

\section{Long-term benefits of LVRS}

In addition to improved mortality in select patients and an improvement in symptoms, patients with upperlobe predominant emphysema who undergo LVRS have shown improvement in multiple cardiopulmonary metrics. Surgical correction of hyperinflation leads to an increase in tidal volume and minute ventilation (14), as well as a reduction in dynamic hyperinflation $(15,16)$. During exercise, patients are able to obtain a higher maximal heart rate, and increased workload. They are less likely to have a respiratory limitation to exercise, and show decreased breathlessness (14). Cardiac improvement following reduction of hyperinflation has been noted, with postLVRS patients experiencing a decrease in end-expiratory pulmonary capillary wedge pressure (17), increased cardiac index (18), and increase $\mathrm{O}_{2}$ pulse (15).

Improvements in cardiopulmonary mechanics also result in a reduced need for supplemental oxygen (19), as well as a $30 \%$ reduction in exacerbation rate (20). In addition, there is a decrease in resting energy expenditure, decreased respiratory muscle oxygen consumption, and weight gain in patients with respiratory cachexia $(21,22)$.

Given the advanced level of lung disease, there is concern that LVRS would preclude lung transplantation. However, multiple studies have demonstrated the safety and efficacy of lung transplantation following LVRS $(23,24)$. Sequential LVRS followed by lung transplantation has shown an improvement in mortality when compared to lung transplant alone (25).

\section{BLVR}

LVRS has an established role in the care of COPD patients with upper lobe emphysema associated with significant gas trapping and hyperinflation. Despite surgical advances, LVRS comes with considerable morbidity and mortality, up to $5 \%$ in the NETT trial (26). As a result, multiple bronchoscopic techniques have been developed in an attempt to achieve a similar effect without the surgical morbidity. BLVR has been studied using lung sealant, oneway EBVs and endobronchial coils (26). To date, EBVs and coils have proven to be most efficacious in therapeutic trials. These interventions may offer considerable benefits in patients who remain symptomatic on maximal medical therapy but are unlikely to tolerate or benefit from surgical lung volume reduction.

\section{Patient selection: EBVs}

The VENT study compared outcomes in 321 patients with severe emphysema who were randomly assigned to EBV placement or usual care (26). Inclusion criteria stipulated severe heterogeneous emphysema $\left(\mathrm{FEV}_{1}\right.$ of $\left.15-45 \%\right)$ with hyperinflation [total lung capacity (TLC) $>100 \%$ ] and gas trapping [residual volume (RV) $>150 \%$ ]. At 6 months, the patients in the EBV group were found to have a statistically significant improvement in $\mathrm{FEV}_{1}$ (27). However, there was only a $2 \%$ absolute change in $\mathrm{FEV}_{1}$ which was deemed clinically insignificant. Similarly, patients in the intervention group had a statistical improvement in 6-MWD by an absolute difference of $19 \mathrm{~m}$ which was also not clinically significant.

However, subgroup analyses revealed that patients with increasing heterogeneity in the distribution of emphysema and intact fissures had a more profound improvement in $\mathrm{FEV}_{1}$ and 6-MWD. Review of adverse events revealed that these patients had increased COPD exacerbations and hemoptysis in the months following the procedure (27).

In their 2015 study, Davey et al. looked to refine the patient population for EBV therapy (28). They enrolled 50 patients with heterogeneous emphysema and intact fissures in the target lobe in an RCT. In this population, EBV placement conferred a significant improvement in $\mathrm{FEV}_{1}$ (9\% vs. 3\%) and 6-MWD ( 25 vs. $3 \mathrm{~m})$. There was an increase in rate of pneumothorax in the intervention group ( $8 \% v s .4 \%)$ which was attributed to the improved response to therapy leading to more rapid loss of volume in the targeted lobe and more rapid expansion of the non-targeted ipsilateral lobe.

To further refine patient selection, Klooster et al. evaluated patients with severe emphysema and intact 
Table 3 Patient selection for endobronchial valves $v s$. endobronchial coils

\begin{tabular}{lcc}
\hline $\begin{array}{l}\text { Imaging or lung volume } \\
\text { parameter }\end{array}$ & $\begin{array}{c}\text { Endobronchial } \\
\text { valves }\end{array}$ & $\begin{array}{c}\text { Endobronchial } \\
\text { coils }\end{array}$ \\
\hline TLC $>100 \%$ & $\sqrt{ }$ & $\sqrt{ }$ \\
RV $>150 \%$ & $\sqrt{ }$ & - \\
RV $>175 \%$ & $\sqrt{ }$ & $\sqrt{ }$ \\
Heterogenous emphysema & $\sqrt{ }$ & $\sqrt{ }$ \\
Homogeneous emphysema & $\sqrt{ }$ & $\sqrt{ }$ \\
With collateral ventilation & - & $\sqrt{ }$ \\
Without collateral ventilation & $\sqrt{ }$ & $\sqrt{ }$ \\
\hline
\end{tabular}

$\mathrm{RV}$, residual volume; TLC, total lung capacity.

fissures but compared homogeneous and heterogeneous disease (29). Ultimately, the study randomized 68 patients with severe emphysema and without collateral ventilation to EBV placement or usual medical care. They found that patients had clinically significant improvements in lung function and exercise capacity in both heterogeneous and homogeneous emphysema; however, the effect was more pronounced in patients with heterogeneous disease. Similar to the other trials, an increased rate of pneumothorax was present in the patients who had EBV placement.

In a study of purely homogenous emphysema in patients without collateral ventilation as determined by complete fissures, EBV treatment provided clinically significant benefits in $\mathrm{FEV}_{1}, 6-\mathrm{MWD}$, and symptom burden as assessed by SGRQ (30). Additionally, the study showed a $97 \%$ lobar volume reduction in the targeted lobe with a mean volume reduction of over 1.1 L. Again, pneumothorax was noted in a significantly higher proportion of patients undergoing the procedure.

\section{Patient selection: endobronchial coils}

In addition to one-way EBVs, researchers have studied nitinol coils which can be placed via bronchoscopy. The coils range in length from $10-15 \mathrm{~cm}$ and are designed to regain their shape after deployment. The coils are designed to compress emphysematous tissue to improve pulmonary mechanics and elasticity in neighboring lung tissue (31).

In the RENEW trial, over 300 participants with severe emphysema and gas trapping (TLC >100\%, RV >175\%) were randomized to bilateral endobronchial coil placement or usual care. Overall, patients in the intervention group had a modest improvement in exercise tolerance and $\mathrm{FEV}_{1}(7)$. Upon subgroup analysis, patients with RV $>225 \%$ were found to have a more profound improvement in 6-MWD, lung function and quality of life. These results persisted regardless of collateral ventilation or distribution of emphysema (31).

Another multicenter study of 100 patients showed improved exercise tolerance and modest improvement in lung function in patients undergoing bilateral coil placement as compared with usual care (32). In each of these trials patients were found to have increased rates of pneumonia, pneumothorax and acute exacerbation of COPD in the intervention group $(31,32)$.

\section{Additional methods}

One multicenter RCT evaluated thermal vapor ablation as a means to improve lung function and quality of life in patients with severe upper lobe predominant emphysema at 6 and 12 months duration. The most common adverse reaction was increase in acute exacerbation of COPD. This technique has been used minimally in practice due to lack of commercial availability (33).

\section{Survival in BLVR}

Though none of the large, RCTs were powered to show a mortality benefit with BLVR, a few smaller retrospective reviews have analyzed this outcome in patients undergoing BLVR. In an analysis of 19 patients who underwent EBV placement, patients who had significant atelectasis on CT scan at 1 -month post procedure had improved survival at 10 years when compared with patients whose scans did not demonstrate atelectasis (34). In a similar analysis Gompelmann and colleagues found a survival benefit at 5 years in patient with atelectasis after EBV placement (35). Though small and lacking a control group these analyses provide some evidence that successful BLVR may confer a survival benefit $(34,35)$.

\section{Summary}

In summary, EBV or coil placement has proven to be effective in improving exercise tolerance and lung function in patients with severe emphysema, gas trapping and hyperinflation. For EBVs, studies have shown that these benefits occur in a relatively broad patient population with heterogeneous or homogeneous emphysema provided patients do not have collateral ventilation. Endobronchial coils have shown somewhat more modest improvements but benefits persist despite the presence of collateral ventilation (Table 3). However, when compared with usual 


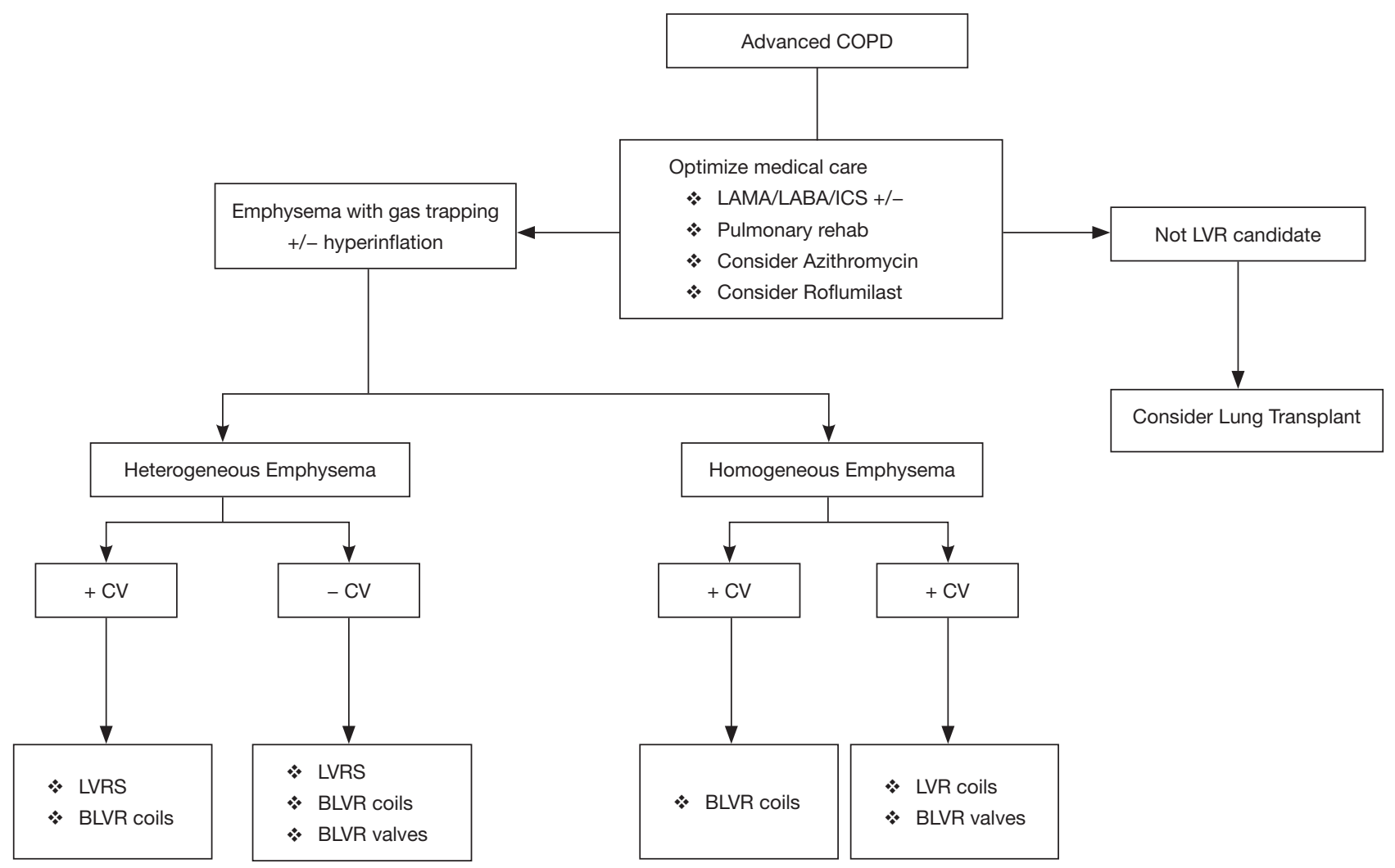

Figure 1 Therapeutic pathway in advanced COPD. Adapted from 2017 report of Global Initiative for Chronic Obstructive Lung Disease (GOLD) (36). COPD, chronic obstructive pulmonary disease; LAMA, long acting muscarinic antagonists; LABA, long acting beta agonists; ICS, inhaled corticosteroids.

medical care, EBV or coil placement confers higher risk of pneumothorax, hemoptysis and COPD exacerbation in the peri-procedural period (27-33).

\section{Conclusions}

In patients with COPD, hyperinflation is associated with significant cardiopulmonary impairment, increased dyspnea, and reduced exercise tolerance. Reduction in hyperinflation via lung volume reduction has shown remarkable improvements in both mortality and symptoms. However, these improvements are limited to a carefully selected group of patients. For optimal outcomes, phenotyping is necessary.

For patients who qualify for LVRS, this procedure remains the only therapy with a proven mortality benefit and durable improvement in symptoms, albeit at the cost of increased peri-operative morbidity. In patients who qualify, LVRS should be considered the Global Initiative for Chronic Obstructive Lung Disease (GOLD) standard. In patients who do not meet the NETT criteria or are unwilling to undergo surgery, minimally invasive techniques should be considered. In patients with intact fissures and upper lobe predominant disease, both EBVs and coils have shown improvement in $\mathrm{FEV}_{1}$ and 6-MWD. Further phenotyping is necessary, as patients without collateral ventilation have shown improvement with both coils and valves, while those with collateral ventilation do not benefit from EBV therapy (Figure 1) (36).

\section{Acknowledgements}

None.

\section{Footnote}

Conflicts of Interest: The authors have no conflicts of interest to declare. 


\section{References}

1. Adeloye D, Chua S, Lee C, et al. Global and regional estimates of COPD prevalence: Systematic review and meta-analysis. J Glob Health 2015;5:020415.

2. World Health Organization. Chronic obstructive pulmonary disease (COPD). Available online: http://www. who.int/respiratory/copd/en

3. Anthonisen NR, Connett JE, Murray RP. Smoking and lung function of Lung Health Study participants after 11 years. Am J Respir Crit Care Med 2002;166:675-9.

4. Stuart-Harris C, D. C. Flenley, and J. H. Bishop. Long term domiciliary oxygen therapy in chronic hypoxic cor pulmonale complicating chronic bronchitis and emphysema. Report of the Medical Research Council Working Party. Lancet 1981;1:681-6.

5. Nocturnal Oxygen Therapy Trial Group. Continuous or nocturnal oxygen therapy in hypoxemic chronic obstructive lung disease: a clinical trial. Nocturnal Oxygen Therapy Trial Group. Ann Intern Med 1980;93:391-8.

6. Fishman A, Martinez F, Naunheim K, et al. A randomized trial comparing lung-volume-reduction surgery with medical therapy for severe emphysema. N Engl J Med 2003;348:2059-73.

7. Part B National Summary Data File (Previously known as BESS). Available online: https://www.cms.gov/ResearchStatistics-Data-and-Systems/Downloadable-Public-UseFiles/Part-B-National-Summary-Data-File/Overview.html

8. Brantigan OC, Mueller E, Kress MB. A surgical approach to pulmonary emphysema. Am Rev Respir Dis 1959;80:194-206.

9. Cooper JD, Trulock EP, Triantafillou AN, et al. Bilateral pneumectomy (volume reduction) for chronic obstructive pulmonary disease. J Thorac Cardiovasc Surg 1995;109:106-16; discussion 116-9.

10. Cooper JD, Patterson GA, Sundaresan RS, et al. Results of 150 consecutive bilateral lung volume reduction procedures in patients with severe emphysema. J Thorac Cardiovasc Surg 1996;112:1319-29; discussion 1329-30.

11. Criner GJ, Cordova FC, Furukawa S, et al. Prospective randomized trial comparing bilateral lung volume reduction surgery to pulmonary rehabilitation in severe chronic obstructive pulmonary disease. Am J Respir Crit Care Med 1999;160:2018-27.

12. Geddes D, Davies M, Koyama H, et al. Effect of lungvolume-reduction surgery in patients with severe emphysema. N Engl J Med 2000;343:239-45.

13. Chandra D, Lipson DA, Hoffman EA, et al. Perfusion scintigraphy and patient selection for lung volume reduction surgery. Am J Respir Crit Care Med 2010;182:937-46.

14. Criner GJ, Belt P, Sternberg AL, et al. Effects of lung volume reduction surgery on gas exchange and breathing pattern during maximum exercise. Chest 2009;135:1268-79.

15. Lammi MR, Ciccolella D, Marchetti N, et al. Increased oxygen pulse after lung volume reduction surgery is associated with reduced dynamic hyperinflation. Eur Respir J 2012;40:837-43.

16. Martinez FJ, de Oca MM, Whyte RI, et al. Lung-volume reduction improves dyspnea, dynamic hyperinflation, and respiratory muscle function. Am J Respir Crit Care Med 1997;155:1984-90.

17. Criner GJ, Scharf SM, Falk JA, et al. Effect of lung volume reduction surgery on resting pulmonary hemodynamics in severe emphysema. Am J Respir Crit Care Med 2007;176:253-60.

18. Jorgensen K, Houltz E, Westfelt U, et al. Effects of lung volume reduction surgery on left ventricular diastolic filling and dimensions in patients with severe emphysema. Chest 2003;124:1863-70.

19. Snyder ML, Goss CH, Neradilek B, et al. Changes in arterial oxygenation and self-reported oxygen use after lung volume reduction surgery. Am J Respir Crit Care Med 2008;178:339-45.

20. Washko GR, Fan VS, Ramsey SD, et al. The effect of lung volume reduction surgery on chronic obstructive pulmonary disease exacerbations. Am J Respir Crit Care Med 2008;177:164-9.

21. Vestbo J, Prescott E, Almdal T, et al. Body mass, fatfree body mass, and prognosis in patients with chronic obstructive pulmonary disease from a random population sample: findings from the Copenhagen City Heart Study. Am J Respir Crit Care Med 2006;173:79-83.

22. Mineo TC, Pompeo E, Mineo D, et al. Resting energy expenditure and metabolic changes after lung volume reduction surgery for emphysema. Ann Thorac Surg 2006;82:1205-11.

23. Orens JB, Estenne M, Arcasoy S, et al. International Guidelines for the Selection of Lung Transplant Candidates: 2006 Update-A Consensus Report From the Pulmonary Scientific Council of the International Society for Heart and Lung Transplantation. J Heart Lung Transplant 2006;25:745-55.

24. Shigemura N, Gilbert S, Bhama JK, et al. Lung transplantation after lung volume reduction surgery. 
Transplantation 2013;96:421-5.

25. Weinstein MS, Martin UJ, Crookshank AD, et al. Mortality and functional performance in severe emphysema after lung volume reduction or transplant. COPD 2007;4:15-22.

26. Criner GJ, Cordova F, Sternberg AL, et al. The National Emphysema Treatment Trial (NETT) Part II: Lessons learned about lung volume reduction surgery. Am J Respir Crit Care Med 2011;184:881-93.

27. Sciurba FC, Ernst A, Herth FJ, et al. A randomized study of endobronchial valves for advanced emphysema. N Engl J Med 2010;363:1233-44.

28. Davey C, Zoumot Z, Jordan S, et al. Bronchoscopic lung volume reduction with endobronchial valves for patients with heterogeneous emphysema and intact interlobar fissures (the BeLieVeR-HIFi study): a randomised controlled trial. Lancet 2015;386:1066-73.

29. Klooster K, ten Hacken NH, Hartman JE, et al. Endobronchial Valves for Emphysema without Interlobar Collateral Ventilation. N Engl J Med 2015;373:2325-35.

30. Valipour A, Slebos DJ, Herth F, et al. Endobronchial Valve Therapy in Patients with Homogeneous Emphysema. Results from the IMPACT Study. Am J Respir Crit Care Med 2016;194:1073-82.

Cite this article as: Gordon M, Duffy S, Criner GJ. Lung volume reduction surgery or bronchoscopic lung volume reduction: is there an algorithm for allocation? J Thorac Dis 2018;10(Suppl 23):S2816-S2823. doi: 10.21037/jtd.2018.05.118
31. Sciurba FC, Criner GJ, Strange C, et al. Effect of Endobronchial Coils vs Usual Care on Exercise Tolerance in Patients With Severe Emphysema: The RENEW Randomized Clinical Trial. JAMA 2016;315:2178-89.

32. Deslee G, Mal H, Dutau H, et al. Lung Volume Reduction Coil Treatment vs Usual Care in Patients With Severe Emphysema: The REVOLENS Randomized Clinical Trial. JAMA 2016;315:175-84.

33. Herth FJ, Valipour A, Shah PL, et al. Segmental volume reduction using thermal vapour ablation in patients with severe emphysema: 6-month results of the multicentre, parallel-group, open-label, randomised controlled STEPUP trial. Lancet Respir Med 2016;4:185-93.

34. Garner J, Kemp SV, Toma TP, et al. Survival after Endobronchial Valve Placement for Emphysema: A 10-Year Follow-up Study. Am J Respir Crit Care Med 2016;194:519-21.

35. Gompelmann D, Benjamin N, Bischoff E, et al. Survival after endoscopic valve therapy in patients with severe emphysema. Eur Respir J 2016;48:OA472.

36. GOLD 2017 Global Strategy for the Diagnosis, Management and Prevention of COPD. Available online: http://goldcopd.org/gold-2017-global-strategy-diagnosismanagement-prevention-copd/ 